\title{
Electromagnetic Toroidal Excitations in Matter and Free Space
}

\author{
N. Papasimakis ${ }^{1, *}$, V. A. Fedotov ${ }^{1}$, V. Savinov ${ }^{1}$, T. A. Raybould ${ }^{1}$, and N. I. Zheludev ${ }^{1,2}$ \\ ${ }^{1}$ Optoelectronics Research Centre \& Centre for Photonic Metamaterials, \\ University of Southampton, Highfield SO17 1BJ, UK \\ ${ }^{2}$ TPI and Centre for Disruptive Photonic Technologies, \\ Nanyang Technological University, Singapore 637378, Singapore \\ n.papasimakis@soton.ac.uk
}

The toroidal dipole is a localized electromagnetic excitation, distinct from the magnetic and electric dipoles. While the electric dipole can be understood as a pair of opposite charges and the magnetic dipole as a current loop, the toroidal dipole corresponds to currents flowing on the surface of a torus. Toroidal dipoles provide physically significant contributions to the basic characteristics of matter including absorption, dispersion, and optical activity. Toroidal excitations also exist in free-space as spatially and temporally localized electromagnetic pulses propagating at the speed of light and interacting with matter. We review recent experimental observations of resonant toroidal dipole excitations in metamaterials and the discovery of anapoles, non-radiating current configurations involving toroidal dipoles. While certain fundamental and practical aspects of toroidal electrodynamics remain open for the moment, we envision that exploitation of toroidal response can have important implications for the fields of photonics, sensing, energy and information.

\section{Introduction}

The interactions of electromagnetic radiation with matter underpin some of the most important technologies today - from telecommunications to information processing and data storage; from spectroscopy and imaging to light-assisted manufacturing. Our understanding and description of the electromagnetic properties of matter traditionally involves the concept of electric and magnetic dipoles, as well as their more complex combinations, known as multipoles. Introduced by Maxwell and Lorentz and later refined by Jackson and Landau, this framework, termed the multipole expansion, is central in physics and is being routinely applied in the study of optical, condensed matter, atomic, nuclear phenomena and beyond ${ }^{1}$. Within this framework, electromagnetic media can be represented by a set of point-like multipole sources ${ }^{2-5}$. The commonly used set of multipoles comprises the electric and magnetic families, which can be represented by oscillating charges and loop currents respectively. Dynamic toroidal multipoles constitute a third independent family of elementary electromagnetic sources, rather than an alternative multipole expansion or higher-order corrections to the conventional electric and magnetic multipoles (see tutorial inset I).

Introduced in 1958 by Y. B. Zeldovich, toroidal moments have been considered in systems of toroidal topology (see Fig. 1) and studied in the context of nuclear ${ }^{6}$, atomic ${ }^{7}$, and molecular physics ${ }^{8}$, classical electrodynamics ${ }^{9,10}$, and solid state physics ${ }^{3}$. In the field of electromagnetism, in particular, a number of works have led to the development of a complete theoretical framework for toroidal electrodynamics ${ }^{11-15}$ and the prediction of exotic effects, including dynamic non-radiating charge current configurations ${ }^{10}$ and non-reciprocal interactions ${ }^{9}$. Following recent experimental observations of toroidal contributions in the response of materials across the electromagnetic spectrum, dynamic 
toroidal multipoles are now the focus of substantial research efforts. Here, we will trace the development of toroidal electrodynamics from the early considerations of static toroidal moments in condensed matter, to the recent experimental works on dynamic toroidal multipoles. We will examine the way by which toroidal multipoles enter the description of the materials' response, a topic of ongoing discussion, and review advances in the studies of the peculiar radiation properties of toroidal dipoles, as well as the free-space propagating toroidal excitations.

\section{Static toroidal multipoles}

Initially, the static toroidal dipole (or anapole) was introduced as a configuration of static currents flowing on the surface of a torus ${ }^{17}$, in order to represent nuclear currents that participate in parityviolating weak interactions (see Fig. 1). It was pointed out that the external field of such a configuration is identically zero, whereas the currents create a magnetic field confined within the torus; hence static toroidal dipoles do not interact directly with electric and magnetic fields ${ }^{17}$. The concept of the anapole was generalized to a full family of toroidal multipoles and applied to the description of condensed matter $^{3,18}$. Toroidal ordering in the solid-state was first investigated theoretically in 1946 by Charles Kittel in his work on ferromagnetic domains of small particles ${ }^{19}$ and experimentally in $1974^{20}$, followed by a series of observations, in 1984 and 1985, which confirmed the existence of static toroidal moments ${ }^{21,22}$ (see also ${ }^{23}$ and references therein). Two sets of toroidal multipoles have been considered in this context, termed toroidal electric (or axial) and toroidal magnetic (or polar) ${ }^{2}$. The first set arises from vortex-like configurations of electric dipoles ${ }^{2}$, whereas the latter set arises from currents flowing on the surfaces of tori, along the meridians, or, equivalently, from vortex-like configurations of magnetic dipoles (see Fig. 2). Similarly, the family of electric (magnetic) toroidal multipoles can be generated by magnetic (electric) charge currents. However, since magnetic currents have not been observed to date, static electric toroidal multipoles can only be imitated, by virtue of the dual symmetry, through electric polarization ${ }^{2}$, whereas dynamic electric toroidal multipoles cannot exist. It is for this reason that, from these two sets of toroidal moments, research interest has concentrated predominantly on the magnetic toroidal multipoles. In this Progress Article, electric toroidal multipoles are discussed only in the static regime.

Toroidal multipoles enter the description of condensed matter by an order parameter related to the toroidal dipole moment, termed toroidization or toroidal polarization, analogous to the macroscopic electric polarization and magnetization ${ }^{24}$. Where electric polarization accounts for the electric dipole density and magnetization for magnetic dipole density, toroidization represents the density of toroidal dipoles. A macroscopic toroidization emerges from configurations of local toroidal moments that exhibit long-range order ${ }^{25}$. Such media are called ferrotoroids, on equal footing with ferroelectrics and ferromagnets. While ferroelectrics break spatial inversion symmetry and ferromagnets break time reversal symmetry, ferrotoroids with magnetic toroidization break simultaneously time reversal and spatial inversion symmetry. Ferrotoroids with electric toroidization, on the other hand, remain invariant under both time and space inversions ${ }^{26-28}$. Ferrotoroids will exhibit electric polarization (magnetization) in response to an external magnetic (electric) field. This magnetoelectric response is expected to form the basis for applications in technological areas, such as data storage, where for example an electric field can read or write information in the magnetic state of a medium ${ }^{29,30}$. In addition, ferrotoroids are expected to exhibit unique forms of magnetic response ${ }^{31-33}$ and nonreciprocal reflection and dichroism ${ }^{18}$.

Today, the range of static toroidal systems under study includes various compounds ${ }^{34,35}$, boracites ${ }^{36}$, pyroxines $^{37}$ and olivines ${ }^{38}$, metals ${ }^{39}$, glasses ${ }^{40}$, ferroelectric nanoscale disks and rods ${ }^{27}$, molecular magnets ${ }^{41,42}$. However, unambiguous observations of long-range toroidal order have proven challenging due to the weak, short-range interactions between toroidal dipoles and the requirement of simultaneously breaking both space and time reversal symmetry to demonstrate its existence ${ }^{18}$. Early experiments focused on the magnetoelectric effect ${ }^{43,44}$, which is not unique to the toroidal moments ${ }^{28}$, only provided an indication for ferrotoroidic order. Subsequent experiments reported direct observations of ferrotoroidicity, where ferrotoroidic domains were observed in an olivine crystal $\left(\mathrm{LiCoPO}_{4}\right)$ and domain orientations were identified by second harmonic generation ${ }^{26}$. These findings 
were confirmed in follow-up works demonstrating hysteretic effects by a similar nonlinear optical method $^{45}$.

\section{Dynamic toroidal multipoles}

In the dynamic regime, only magnetic toroidal moments can exist, whereas electric toroidal multipoles are absent, as explained in the previous section. Thus, for simplicity we will refer to magnetic toroidal moments as toroidal in this section. Dynamic toroidal dipoles, in contrast to their static counterparts, interact with oscillating electromagnetic fields, and thus contribute to the optical properties of materials across the entire electromagnetic spectrum ${ }^{3}$. However, the inclusion or omission of the dynamic toroidal multipoles from the multipole expansion has been the subject of an on going discussion ${ }^{46-48}$, in particular with respect to equivalent descriptions, such as Mie theory ${ }^{49,50}$. We address this in detail in a dedicated section (see tutorial inset II), where we demonstrate that although both the vector spherical harmonic Mie expansion and the (full, including toroidal contributions) charge-current multipole expansion are complete, the toroidal multipoles appear as distinct entities only in the latter. This has direct implications for the interpretation of electromagnetic excitations and optical forces in media of toroidal topology and, in combination with the experimental observations of isolated toroidal dipoles, justifies the consideration of toroidal moments as a separate family of multipoles.

Although observations of toroidal excitations are often complicated by the contributions of the electric and magnetic multipoles to the material response, they may be expected in the case of large molecules or molecules with toroidal symmetry. Indeed, electromagnetic fields scattered by the toroidal dipole moment of a molecule with a characteristic size R scale with the free-space wavelength $\lambda$ as $\sim(\mathrm{R} / \lambda)^{3}$, while electric and magnetic dipole scattering scales as $\sim(\mathrm{R} / \lambda)$ and $\sim(\mathrm{R} / \lambda)^{2}$, respectively ${ }^{13,15,51}$. Hence, the toroidal response of a system will become increasingly important in comparison to the standard dipoles, as the size of the system or molecule increases. Relatively large molecules with $\mathrm{R} \leq \lambda$, known as "metamolecules" are often employed in artificial metamaterials. A metamaterial of toroidal topology can be engineered in such a way that the lower order electric and magnetic multipoles cannot be excited due to symmetry, allowing thus the toroidal dipole to become the leading term in the multipole expansion and to contribute strongly to the electromagnetic properties of the metamaterial ${ }^{10}$. When scattering contributions of the conventional multipoles are dominant, toroidal response of the nanostructure might still be detected through optical forces ${ }^{50}$. Even for small, deeply sub-wavelength systems, such as atoms and molecules, one can expect that toroidal dipole excitations should be observable, in principle, given that effects of the same order, for instance magnetic quadrupole transitions ${ }^{52}$, are also experimentally accessible.

\section{Toroidal response in artificial media}

Metamaterials, artificial media periodically structured at the sub-wavelength scale in order to achieve desirable electromagnetic functionality, served as a platform for the first observation of resonances due to induced toroidal dipoles. Early works on toroidal metamaterials predicted the presence of backward waves and negative refraction in such material systems ${ }^{53}$. Experimental signatures of a toroidal dipole response were first seen in the microwave dichroism spectra of chiral toroidal solenoid arrays in $2009^{54}$, obscured however by the presence of dipole and higher order electric and magnetic multipoles. First observation of an isolated toroidal dipole absorption resonance was reported in $2010^{55}$ in a metamaterial whose metamolecules were formed by a ring-shaped arrangement of microwave resonators (see Fig. 3a). In this type of metamaterial, excitation at the resonant frequency induces a loop chain of oscillating magnetic dipole moments, the excitation of which is possible due to a combination of retardation effects and electromagnetic interactions between individual resonators. The induced magnetic dipoles trace the circumference of a closed loop leading to a field structure similar to that of a toroidal solenoid. These initial observations were quickly followed by a number of works aiming to further enhance the toroidal response and suppress the contributions of competing electric and magnetic multipoles ${ }^{56,57}$.

Planar designs were considered ${ }^{58,59}$ (Fig. 3b) in an effort to simplify the fabrication of toroidal metamaterials. By scaling down metamaterial designs based on clusters of split-ring resonators, toroidal 
response at terahertz ${ }^{60}$ and optical frequencies ${ }^{61,62}$ (Fig. 3c) has been unambiguously detected. In parallel, fabrication difficulties were also overcome by introducing artificial patterns that are less challenging to manufacturing at the nanoscale, where split-ring resonators are replaced by pairs of bars ${ }^{63}$ (Fig. 3d) and disks ${ }^{64,65}$, while still supporting toroidal excitation modes. In the optical part of the spectrum, a toroidal dipole response, although weakened due to high ohmic losses in metals, was found in even simpler systems, such as plasmonic core-shell nanoparticles ${ }^{66}$, and bas-relief patterns that support spoof plasmons, including periodic grids ${ }^{67}$ and arrays of ring-shaped grooves illuminated at oblique angles $^{68}$ (Fig. 3e). A toroidal response was also observed in plasmonic void oligomers using energy-loss transmission electron microscopy ${ }^{69}$ (Fig. 3f). Finally, recent studies of toroidal excitations moved towards novel and active laser emitters ${ }^{70}$, and low-loss dielectric ${ }^{71}$ (Fig. 3g) and superconducting ${ }^{59}$ material systems.

The link between the dynamic microscopic toroidal response and directly observable macroscopic quantities (such as material transmission, reflection and absorption), has been investigated in a series of works ${ }^{24,25,48,51,72}$, but the discussion on the form of the constitutive equations which include the toroidal response remains open.

\section{Radiating properties of toroidal multipoles}

The recent observations of toroidal dipole excitations in metamaterials have enabled the study of their peculiar radiation properties. The radiation pattern of the toroidal dipole is identical to that of an electric dipole (apart from a phase factor), although the corresponding charge-current configurations are different (see Fig. 2). Hence, a coherent superposition of dynamic electric and toroidal dipoles can be realized in a way that the radiated fields by the two dipoles interfere destructively ${ }^{10,14,15}$. Such a nonradiating configuration is in fact a dynamic version of the anapole; in contrast to the static anapole (that directly identifies with the static toroidal dipole), it comprises an electric dipole component ${ }^{24}$. The dynamic anapole was first observed using a microwave metamaterial ${ }^{73}$ (Fig. 3h), where it was shown that the destructive interference between coherently oscillating electric and toroidal dipoles provides a new mechanism of electromagnetic transparency that yields narrow and symmetric Lorentzian transparency lines. Similar resonances were recently detected in the scattering spectra of dielectric nanoparticles ${ }^{49}$ (Fig. 3i) and were also predicted for core-shell wires ${ }^{74}$ and hybrid nanoparticles ${ }^{66}$. Computational studies showed that inhomogeneous dielectric environment perturbs the non-radiating charge-current configuration leading to directional emission. ${ }^{75}$

In addition, non-radiating configurations composed of electric and toroidal dipoles can act as sources of propagating electromagnetic potentials ${ }^{13}$ (see Fig. 4), with the potential as a new channel for information transfer in time-dependent Aharonov-Bohm-like experiments. These suggestions were later challenged; it was argued that not only the fields but also the associated potentials are unobservable everywhere exterior to the spatially localized non-radiating source, while Aharonov-Bohm-type effects associated with non-radiating potentials are only possible in static situations ${ }^{76}$.

Recently, the weak interaction of non-radiating configurations and static toroidal moments with electromagnetic fields has been discussed as a potential mechanism that can protect superconducting qubits from environmental disturbances ${ }^{77}$. Combinations of electric and magnetic moments with their toroidal counterparts give rise to a new mechanism of optical activity, a phenomenon of polarization rotation of electromagnetic waves travelling through chiral media. In the past optical activity was linked to the presence of the electric and magnetic dipole (or electric quadrupole) moments in the medium ${ }^{1}$. Following early work that indicated the toroidal moment contribution to optical activity ${ }^{54}$, a recent experimental demonstration showed a dominant role for the toroidal moment in the polarization properties of a purposely designed microwave metamaterial ${ }^{78}$.

Finally, it has been argued that electrodynamic interactions between toroidal sources and electric or magnetic sources could violate the standard (Lorentz and Feld-Tai) formulation of reciprocity lemmas, under the condition that space- and time-dependence of the two sources cannot be separated or that they do not share the same form of time dependence ${ }^{9}$. These conclusions were extracted by considering the interactions between currents in a toroidal coil and in a ring with different time-dependence, a configuration that breaks action-reaction equality. However, we are not aware of any independent 
verification of this result and to our knowledge these predictions have not been confirmed experimentally; their implications remain unclear to date.

\section{Propagating toroidal excitations}

Although most considerations of toroidal electrodynamics focused on the localized toroidal excitations of matter, a new form of free-space propagating toroidal excitation was predicted ${ }^{79}$ in 1996 and has recently began to receive attention ${ }^{80,81}$. Known as "Focused Doughnuts", such pulses belong to a broader family of finite energy, non-pathological solutions to Maxwell's equations introduced a few years earlier ${ }^{82}$. Focused doughnuts are single-cycle, broad bandwidth pulses with a spatially localized toroidal field configuration and strong longitudinal field components. Importantly, the space and time dependence of such pulses cannot be separated, resulting in a spatially dependent frequency spectrum, in which lower (higher) frequency components dominate the outer (inner) area of the torus. Focused doughnut pulses come in two forms, transverse electric (TE) and transverse magnetic (TM), where one can be obtained from the other by merely exchanging electric and magnetic fields. Although most of the energy of the pulse is confined inside a torus-shaped region (see Fig. 5), the pulses exhibit strong longitudinal field components ${ }^{79}$. In the context of toroidal electrodynamics, it shall be noted that the focused doughnuts have magnetic fields oriented along the equatorial lines of the torus (transverse to the propagation direction) and the electric fields along its meridians, a configuration identical to the one found in the near-field of toroidal resonators. Hence, focused doughnut pulses are essentially free-space propagating versions of the localized toroidal excitations. It was recently reported that focused doughnut pulses can excite dominant toroidal dipoles in a spherical dielectric nanoparticle, a system which lacks toroidal topology ${ }^{81}$. It has been further predicted that the TM pulse exhibits favorable coupling to nanoparticles with a toroidal topology, exciting a broad spectrum of dominant toroidal dipole resonances within a dielectric nanotorus ${ }^{80}$. To date, Focused Doughnut pulses have not yet been realized experimentally, mainly due to the challenging task of controlling simultaneously frequency and spatial dispersion over a wide bandwidth. However, early works suggested the potential of ultra-broadband antenna arrays ${ }^{83}$ for the generation of a broad class of space-time non-separable pulses.

\section{Outlook}

The observation of a resonant toroidal response in metamaterials has enabled the systematic study of toroidal electrodynamics. Despite the recent stream of experimental and theoretical works, however, the field is still in its infancy with many questions to be resolved and applications to be explored. Toroidal resonances in natural media remain to be observed and the spectroscopy of toroidal resonances to be developed. The similarity between the radiation properties of electric and toroidal dipoles calls for a re-examination of spectroscopic data, especially where systems of toroidal symmetry are involved, as is often the case with biological macromolecules ${ }^{84}$.

The full practical potential of natural and artificial media with toroidal elements in their structure is still to be identified; however it is already clear that they interact with electromagnetic waves in an unusual way. While strong toroidal contribution is expected only from large toroidal "molecules" (comparable with the wavelength of light), this contribution can be profound. Toroidal resonances can destructively interfere with other modes of excitations in the materials providing a new mechanism of induced transparency (slow light) and scattering suppression ${ }^{49,73}$ that can be used in narrow-band filters and for controlling emission. Toroidal metamaterials provide useful platform for tailoring electromagnetic environment of complex symmetry and topology for light confinement, trapping and sensor applications (highly gradient electric filed is strong in the centre of the torus). Novel laser designs have been investigated where arrays of artificial toroidal "metamolecules" are uses as gain medium on the frequency of high-Q toroidal mode ${ }^{70}$. An emerging field of study is interaction of toroidal media with structured illumination: for instance toroidal "molecules" efficiently interact with vortex electromagnetic beams ${ }^{85,86}$. Finally, the "Focused Doughnut" pulses as propagating toroidal excitations 
represent an exciting new opportunity for energy and information transfer and, in fact, it is has already been shown that they couple efficiently to toroidal antennas which may be used for their detection ${ }^{80}$.

\section{Tutorial Insets}

\section{Inset I: Toroidal multipoles}

Dynamic toroidal multipoles constitute an independent family of elementary electromagnetic sources. In the absence of intrinsic magnetisation, toroidal multipoles complete the standard dynamic multipole expansion comprising the conventional electric and magnetic multipoles (see Fig. 2). More specifically, they represent the scattering contributions of oscillating radial currents $\boldsymbol{J}$ (that is $\boldsymbol{r} \cdot \boldsymbol{J} \neq 0$ ) and hence are different in nature from the electric and magnetic multipoles associated with oscillating charge density and transverse currents $(\boldsymbol{r} \times \boldsymbol{J} \neq 0)$, respectively. In particular, the lowest order toroidal multipole, the toroidal dipole, corresponds to currents flowing on the surface of an imaginary torus, along its meridians (see Fig. 2). Its moment $\boldsymbol{T}$ is directed along the axis of the torus and is given by $y^{3,15}$

$$
\boldsymbol{T}=\frac{1}{10 c} \int d^{3} r\left[\boldsymbol{r}(\boldsymbol{r} \cdot \boldsymbol{J})-2 r^{2} \boldsymbol{J}\right]
$$

Importantly, the toroidal dipole features neither charge poles (since it is a purely current excitation), nor magnetic poles where magnetic field lines would diverge or converge. Furthermore, the toroidal dipole interacts directly only with external conduction $\left(\boldsymbol{J}_{\text {ext }}\right)$ and displacement currents $(\partial \boldsymbol{D} / \partial \mathrm{t})$ or, equivalently, with a vortex of magnetic field (curl $\boldsymbol{B})$, as it follows from the expression for the energy of its interaction with electromagnetic fields (for a comparison with the electric and magnetic dipole terms, see Table 1$)^{11}$

$$
W_{T}=-\boldsymbol{T} \cdot\left(\frac{4 \pi}{c} \boldsymbol{J}_{\text {ext }}+\frac{1}{c} \frac{\partial \boldsymbol{D}}{\partial t}\right) .
$$

In particular, in the static case, the toroidal dipole interacts only with conduction currents, and tends to align its moment parallel to the current flow.

Despite being physically distinct from the electric dipole, the toroidal dipole emits radiation with the same angular momentum and parity properties as the former ${ }^{5,13}$, and therefore the two multipoles cannot be distinguished by a distant observer. Nevertheless, the toroidal dipole emission presents a distinct frequency dependence, and in the harmonic case where $\boldsymbol{T}(t) \propto e^{i \omega t}$, its intensity has an additional scaling factor $k^{2}=\omega^{2} / c^{2}$ as compared to the electric dipole $e^{15}$ :

$$
I_{T}=\frac{2}{3} c k^{4}|k \boldsymbol{T}|^{2},
$$

which, in principle, allows one to disentangle toroidal and electric dipole emissions via spectroscopic analysis ${ }^{5,15}$. Moreover, the vector potential, $\boldsymbol{A}_{T}(r, t)$, of the toroidal emission differs from the vector potential, $\boldsymbol{A}_{p}(r, t)$, emitted by an electric dipole, and, in fact, their difference $\Delta \boldsymbol{A}=\boldsymbol{A}_{T}-\boldsymbol{A}_{p}$ cannot be eliminated via a gauge transformation ${ }^{13}$. Indeed, the electromagnetic fields emitted by a point-like toroidal dipole, superimposed with a point-like electric dipole $\boldsymbol{p}=i k \boldsymbol{T}$ vanish at any distance away from the source (but not at the source), whereas for the corresponding vector potential remains nonzero ${ }^{51}$ 


$$
\Delta \boldsymbol{A}=\nabla\left(\boldsymbol{T} \cdot \nabla\left(\frac{e^{-i k r}}{r}\right)\right)+4 \pi \delta^{3}(r) \boldsymbol{T}
$$

Since all effects of gauge-fixing can be described by addition of a gradient of an arbitrary function, $\nabla f$, to the vector potential, the non-trivial part of the vector potential, irremovable by gauge-fixing, will be given by terms with non-zero curl:

$$
\nabla \times(\Delta \boldsymbol{A})=\nabla \times\left[\nabla\left(\boldsymbol{T} \cdot \nabla\left(\frac{e^{-i k r}}{r}\right)\right)\right]+4 \pi\left[\nabla \delta^{3}(r) \times \boldsymbol{T}\right]
$$

It follows that the second term in the expression for the net vector potential does not vanish upon application of the curl, which indicates that net vector potential cannot be eliminated at all points in space in any gauge, and is therefore non-trivial.

We would like to note that in the current and following insets, we employ a Gaussian unit system, in accordance with the majority of works on the topic of toroidal electrodynamics. Simple means to convert the relations reported here to SI units can be found in ${ }^{89}$.

\section{Inset II: Charge -current multipole expansion vs. Mie theory}

Both charge-current multipole expansion and Mie theory provide a complete mathematical description of electromagnetic scattering in the far field. However, toroidal excitations seemingly appear only in the multipole expansion. This apparent discrepancy is explained in this inset.

Multipole expansion. The multipole expansion (if carried out in the spherical basis) represents scattered electromagnetic field as a series of vector spherical wave harmonics $\boldsymbol{\Psi}_{l, m}$ and $\boldsymbol{\Phi}_{l, m}$ (where $\boldsymbol{\Psi}_{l, m}=-i\left[\nabla \times \boldsymbol{\Phi}_{l, m}\right] / k$ ), with coefficients that in the absence of magnetization are explicitly determined by radial and angular distributions of charge $\rho$ and current $\mathbf{J}$ densities induced in the scatterer:

$$
\begin{aligned}
& \mathbf{E}_{\text {sca }}=\frac{4 \pi k^{2}}{c} \sum_{l, m}\left(Q_{l, m} \mathbf{\Psi}_{l, m}+M_{l, m} \mathbf{\Phi}_{l, m}+T_{l, m} \mathbf{\Psi}_{l, m}\right) \\
& Q_{l, m}=\frac{c}{i \sqrt{l(l+1)}} \int \rho Y_{l, m}^{*} \frac{d}{d r}\left[r j_{l}(k r)\right] d^{3} r \\
& M_{l, m}=\frac{1}{i \sqrt{l(l+1)}} \int(\nabla \cdot[\mathbf{r} \times \mathbf{J}]) Y_{l, m}^{*} j_{l}(k r) d^{3} r \\
& T_{l, m}=\frac{k}{\sqrt{l(l+1)}} \int(\mathbf{r} \cdot \mathbf{J}) Y_{l, m}^{*} j_{l}(k r) d^{3} r
\end{aligned}
$$

These expansion coefficients $Q_{l, m}, T_{l, m}$, and $M_{l, m}$, known as multipole moments, characterize the strength of dynamic multipoles - modes of localized charge and current oscillations that replace the actual distributions of $\rho$ and $\boldsymbol{J}$, and act as elementary point-like sources of spherical wave harmonics. Such sources are represented by three families of physically independent excitations, which correspond to volumetric oscillations of (i) charges yielding electric multipoles, (ii) transverse currents $(\boldsymbol{r} \times \boldsymbol{J} \neq 0)$ 
yielding magnetic multipoles, and (iii) radial currents $(\boldsymbol{r} \cdot \boldsymbol{J} \neq 0$ ) yielding toroidal multipoles. In general, sources of all three types contribute to electromagnetic scattering and their contributions can be uniquely identified through the multipole moments ${ }^{3,5,15}$.

Mie theory. In the frame of Mie theory the spherical harmonic expansion is applied to scattered, as well as incident and internal (with respect to the scatterer) electromagnetic fields. While the expansion coefficients for the incident fields are given, those for the internal and scattered fields (known as Mie coefficients) are determined by enforcing continuity of the fields across the scatterer's boundary. Since the scattered magnetic field has no radial component, while the radial component of electric field vanishes in the farfield zone ${ }^{90}$, the boundary conditions are respected only for the transverse components:

$$
\begin{aligned}
& {\left[\left(\mathbf{E}_{\mathrm{inc}}+\mathbf{E}_{\mathrm{sca}}-\mathbf{E}_{\mathrm{int}}\right) \times \mathbf{r}\right]=0,} \\
& {\left[\left(\mathbf{H}_{\mathrm{inc}}+\mathbf{H}_{\mathrm{sca}}-\mathbf{H}_{\mathrm{int}}\right) \times \mathbf{r}\right]=0 .}
\end{aligned}
$$

Correspondingly, Mie expansion features scattering contributions of only two kinds, as defined by the resulting two sets of Mie scattering coefficients $a_{l, m}$ and $b_{l, m}{ }^{90}$

$$
\mathbf{E}_{\mathrm{sca}}=k^{2} \sum_{l, m}\left(A_{l, m} a_{l, m} \boldsymbol{\Psi}_{l, m}+B_{l, m} b_{l, m} \boldsymbol{\Phi}_{l, m}\right),
$$

where $A_{l, m}$ and $B_{l, m}$ are the expansion coefficients for the incident field. Although these contributions are commonly referred to as electric and magnetic multipole fields, $a_{l, m}$ and $b_{l, m}$ are not related (at least directly) to a particular mode of charge-current excitation, but rather to the shape of the scatterer and the spatial structure of scattered electromagnetic field. For example, in the case of a spherical dielectric particle in air with a radius $r_{0}$ and refractive index $n$, the coefficients are defined simply through the spherical Bessel functions $j$ :

$$
\begin{aligned}
& a_{l}=\frac{n^{2} j_{l}\left(n k r_{0}\right)\left[k r_{0} j_{l}\left(k r_{0}\right)\right]^{\prime}-j_{l}\left(k r_{0}\right)\left[n k r_{0} j_{l}\left(n k r_{0}\right)\right]^{\prime}}{n^{2} j_{l}\left(n k r_{0}\right)\left[k r_{0} h_{l}\left(k r_{0}\right)\right]^{\prime}-h_{l}\left(k r_{0}\right)\left[n k r_{0} j_{l}\left(n k r_{0}\right)\right]^{\prime}}, \\
& b_{l}=\frac{j_{l}\left(n k r_{0}\right)\left[k r_{0} j_{l}\left(k r_{0}\right)\right]^{\prime}-j_{l}\left(k r_{0}\right)\left[n k r_{0} j_{l}\left(n k r_{0}\right)\right]^{\prime}}{j_{l}\left(n k r_{0}\right)\left[k r_{0} h_{l}\left(k r_{0}\right)\right]^{\prime}-h_{l}\left(k r_{0}\right)\left[n k r_{0} j_{l}\left(n k r_{0}\right)\right]^{\prime}} .
\end{aligned}
$$

Importantly, since toroidal and electric multipoles are identical in terms of far-field scattering and their difference in the near field (due to non-vanishing radial electric field) is not recognized by the boundary conditions, their contributions in $a_{l, m}$ are mixed together and cannot be separated without the knowledge of the actual charge-current distribution, yielding the so-called renormalized electric multipoles ${ }^{50}$. Thus, unlike the multipole expansion, Mie theory offers merely a mathematical description of the scattering problem providing little physical insight. Consequently, one should exercise caution while establishing a relation between the radiation properties of an electromagnetic scatterer and the distribution of the actual charge-current density (polarization) induced in the scatterer.

Failure to recognize toroidal multipoles in the frame of Mie theory may lead to a confusion and incorrect physical interpretation of the scattering 
phenomena. In particular, such a situation arises in conjunction with a nontrivial non-radiating excitation, also known as a dynamic anapole, where collocated electric and toroidal dipolar modes interfere destructively cancelling each other's radiation everywhere in the far field ${ }^{10,73}$. Since electric dipole scattering in this case is virtually absent the corresponding Mie coefficient $a_{1, m}$ will be zero. The latter suggests that the polarization induced in the scatterer should be also zero, while $a$ priori it is not. This paradox has been recently brought to light by Miroshnichenko and co-workers, who experimentally studied light scattering by Si nanodisks in the visible part of the spectrum ${ }^{49}$. Ignoring the role of the toroidal multipoles in the physical picture may also have implications for understanding the optical force as a consequence of external fields interacting with nanostructures ${ }^{50}$.

\section{Acknowledgements}

The authors are grateful to Din Ping Tsai, Ian Youngs and Janne Ruostekoski for fruitful discussions and acknowledge the support of the MOE Singapore (grant MOE2011-T3-1-005), the UK's Engineering and Physical Sciences Research Council (grants EP/G060363/1, EP/M008797/1), the Defence Science and Technology Laboratory (grant DSTLX-1000068886), and the Leverhulme Trust.

\section{Author's contributions}

All authors made equal contribution to the review and cross-edited the text and figures.

\section{Additional information}

The authors declare no competing financial interests. Reprints and permission information is available online at www.nature.com/reprints. Correspondence and requests for materials should be addressed to N.P. and N.I.Z. The data from this paper can be obtained from the University of Southampton ePrints research repository: http://dx.doi.org/10.5258/SOTON/385194.

\section{References}

1. Raab, R. E. \& de Lange, O. L. Multipole Theory in Electromagnetism. (Oxford University Press, 2005). doi:10.1093/acprof:oso/9780198567271.001.0001

2. Dubovik, V. M., Tosunyan, L. A. \& Tugushev, V. V. Axial toroidal moments in electrodynamics and solid-state physics. J. Exp. Theor. Phys. 63, 344-351 (1986).

3. Dubovik, V. M. \& Tugushev, V. V. Toroid moments in electrodynamics and solid-state physics. Phys. Rep. 187, 145-202 (1990).

4. Vrejoiu, C. Electromagnetic multipoles in Cartesian coordinates. J. Phys. A. Math. Gen. 35, 9911 (2002)

5. Gongora, A. T. \& Ley-Koo, E. Complete electromagnetic multipole expansion including toroidal moments. Rev. Mex. Física 52, 188-197 (2006).

6. Flambaum, V. V. \& Murray, D. W. Anapole moment and nucleon weak interactions. Phys. Rev. C 56, 1641-1644 (1997).

7. Flambaum, V. V. \& Khriplovich, I. B. P-odd nuclear forces - a source of parity violation in atoms. J. Exp. Theor. Phys. 52, 835-839 (1980).

8. Ceulemans, A. \& Chibotaru, L. F. Molecular Anapole Moments. Phys. Rev. Lett. 80, 1861-1864 (1998).

9. Afanasiev, G. N. Simplest sources of electromagnetic fields as a tool for testing the reciprocity-like theorems. $J$. Phys. D. Appl. Phys. 34, 539 (2001). 
10. Afanasiev, G. N. \& Dubovik, V. M. Some remarkable charge-current configurations. Phys. Part. Nucl. 29, 366-391 (1998).

11. Dubovik, V. M. \& Cheshkov, A. A. Multipole current expansion in classic and quantum field theory and radiation. Phys. Elem. Part. Nuclei. 5, 791-837 (1974).

12. Afanasiev, G. N. The electromagnetic field of solenoids with time-dependent currents. J. Phys. A. Math. Gen. 23, 5755-5764 (1990).

13. Afanasiev, G. N. \& Stepanovsky, Y. P. The electromagnetic field of elementary time-dependent toroidal sources. $J$. Phys. A. Math. Gen. 28, 4565-4580 (1995).

14. Radescu, E. E. \& Vlad, D. H. Angular momentum loss by a radiating toroidal dipole. Phys. Rev. E 57, 6030-6037 (1998).

15. Radescu, E. E. \& Vaman, G. Exact calculation of the angular momentum loss, recoil force, and radiation intensity for an arbitrary source in terms of electric, magnetic, and toroid multipoles. Phys. Rev. E 65, 046609 (2002).

16. Super-toroidal electrodynamics (2004). at <http://eprints.soton.ac.uk/id/eprint/385206>

17. Zel’Dovich, I. B. Electromagnetic Interaction with Parity Violation. Sov. J. Exp. Theor. Phys. 6, 1184-1186 (1958).

18. Spaldin, N. A., Fiebig, M. \& Mostovoy, M. The toroidal moment in condensed-matter physics and its relation to the magnetoelectric effect. J. Phys. Condens. Matter 20, 434203 (2008).

19. Kittel, C. Theory of the Structure of Ferromagnetic Domains in Films and Small Particles. Phys. Rev. 70, 965-971 (1946).

20. Zheludev, I. S., Perekalina, T. M., Smirnovskaya, E. M., Fonton, S. S. \& Yarmukhamedov, Y. N. Magnetic Properties of Nickel-Boracite Iodide. J. Exp. Theor. Phys. Lett. 20, 129-130 (1974).

21. Ginzburg, V. L., Gorbatsevich, A. A., Kopayev, Y. V. \& Volkov, B. A. On the problem of superdiamagnetism. Solid State Commun. 50, 339-343 (1984).

22. Sannikov, D. G. \& Zheludev, I. S. The Possibility of Phase Transitions With Spontaneous Toroidal Moment Formation in Nickel Boracites. Sov. Physics-Solid State 27, 826-828 (1985).

23. Crone J.C. Chung, P. W. Modeling of Toroidal Ordering in Ferroelectric Nanodots. Army Res. Lab. report ARLTR-4165 (2007)

24. Dubovik, V. M. Material equations for electromagnetism with toroidal polarizations. Phys. Rev. E 61, 7087-7097 (2000).

25. Ederer, C. \& Spaldin, N. A. Towards a microscopic theory of toroidal moments in bulk periodic crystals. Phys. Rev. B 76, 214404 (2007).

26. Van Aken, B. B., Rivera, J.-P., Schmid, H. \& Fiebig, M. Observation of ferrotoroidic domains. Nature 449, 702705 (2007).

27. Naumov, I. I., Bellaiche, L. \& Fu, H. Unusual phase transitions in ferroelectric nanodisks and nanorods. Nature 432, 737-740 (2004).

28. Planes, A., Castán, T. \& Saxena, A. Recent progress in the thermodynamics of ferrotoroidic materials. Multiferroic Mater. 1, (2015).

29. Khomskii, D. Trend: Classifying multiferroics: Mechanisms and effects. Physics (College. Park. Md). 2, 20 (2009).

30. Pyatakov, A. P. \& Zvezdin, A. K. Magnetoelectric and multiferroic media. Physics-Uspekhi 55, 557-581 (2012).

31. Tolstoi, N. A. \& Spartakov, A. A. Aromagnetism: A new type of magnetism. J. Exp. Theor. Phys. Lett. 52, 161-164 (1990).

32. Fedotov, V. A., Marinov, K., Boardman, A. D. \& Zheludev, N. I. On the aromagnetism and anapole moment of anthracene nanocrystals. New J. Phys. 9, 95 (2007).

33. Martsenyuk, M. A. \& Martsenyuk, N. M. Origin of aromagnetism. J. Exp. Theor. Phys. Lett. 53, $243-246$ (1991).

34. Toledano, P., Khalyavin, D. D. \& Chapon, L. C. Spontaneous toroidal moment and field-induced magnetotoroidic effects in $\mathrm{Ba}_{2} \mathrm{CoGe}_{2} \mathrm{O}_{7}$. Phys. Rev. B 84, 094421 (2011).

35. Tokura, Y. Multiferroics-toward strong coupling between magnetization and polarization in a solid. J. Magn. Magn. Mater. 310, 1145-1150 (2007).

36. Sannikov, D. G. Phenomenological theory of the magnetoelectric effect in some boracites. J. Exp. Theor. Phys. 84, 293-299 (1997). 
37. Mettout, B., Tolédano, P. \& Fiebig, M. Symmetry replication and toroidic effects in the multiferroic pyroxene $\mathrm{NaFeSi}_{2} \mathrm{O}_{6}$. Phys. Rev. B 81, 214417 (2010).

38. Feng, H.-J. \& Liu, F.-M. Ab initio prediction on ferrotoroidic and electronic properties of olivine $\mathrm{Li}_{4}$ MnFeCoNiP $4 \mathrm{O}_{16}$. Chinese Phys. B 18, 2481-2486 (2009).

39. Hayami, S., Kusunose, H. \& Motome, Y. Toroidal order in metals without local inversion symmetry. Phys. Rev. B 90, 024432 (2014).

40. Yamaguchi, Y. \& Kimura, T. Magnetoelectric control of frozen state in a toroidal glass. Nat. Commun. 4, 2063 (2013).

41. Lin, S.-Y. et al. Coupling Dyз Triangles to Maximize the Toroidal Moment. Angew. Chemie Int. Ed. 51, 1276712771 (2012).

42. Berger, R. J. F. Prediction of a Cyclic Helical Oligoacetylene Showing Anapolar Ring Currents in the Magnetic Field. Zeitschrift für Naturforsch. B 67b, 1127-1131 (2012).

43. Popov, Y. F. et al. Magnetoelectric effect and toroidal ordering in $\mathrm{Ga}_{2-\mathrm{x}} \mathrm{Fe}_{\mathrm{x}} \mathrm{O}_{3}$. J. Exp. Theor. Phys. 87, 146-151 (1998).

44. Ressouche, E. et al. Magnetoelectric $\mathrm{MnPS}_{3}$ as a candidate for ferrotoroidicity. Phys. Rev. B 82, 100408 (2010).

45. Zimmermann, A. S., Meier, D. \& Fiebig, M. Ferroic nature of magnetic toroidal order. Nat. Commun. 5, 4796 (2014).

46. Grahn, P., Shevchenko, A. \& Kaivola, M. Electromagnetic multipole theory for optical nanomaterials. New J. Phys. 14, 093033 (2012).

47. Arango, F. B. \& Koenderink, A. F. Polarizability tensor retrieval for magnetic and plasmonic antenna design. New J. Phys. 15, 073023 (2013).

48. Cho, K. Microscopic Expression of Chiral Susceptibilities. in Metamaterials '2011: The Fifth International Congress on Advanced Electromagnetic Materials in Microwaves and Optics (2011).

49. Miroshnichenko, A. E. et al. Nonradiating anapole modes in dielectric nanoparticles. Nat. Commun. 6, 8069 (2015).

50. Zhang, X.-L., Wang, S. B., Lin, Z., Sun, H.-B. \& Chan, C. T. Optical force on toroidal nanostructures: Toroidal dipole versus renormalized electric dipole. Phys. Rev. A 92, 043804 (2015).

51. Savinov, V., Fedotov, V. A. \& Zheludev, N. I. Toroidal dipolar excitation and macroscopic electromagnetic properties of metamaterials. Phys. Rev. B 89, 205112 (2014).

52. Aggarwal K. M. Keenan, F. P. Radiative rates for E1, E2, M1 and M2 transitions in Fe X. Astron. Astrophys. 427, 763-767 (2004).

53. Marinov, K., Boardman, A. D., Fedotov, V. A. \& Zheludev, N. I. Toroidal metamaterial. New J. Phys. 9, 324 (2007).

54. Papasimakis, N., Fedotov, V. A., Marinov, K. \& Zheludev, N. I. Gyrotropy of a Metamolecule: Wire on a Torus. Phys. Rev. Lett. 103, 1 (2009).

55. Kaelberer, T., Fedotov, V. A., Papasimakis, N., Tsai, D. P. \& Zheludev, N. I. Toroidal Dipolar Response in a Metamaterial. Science (80-. ). 330, 1510-1512 (2010).

56. Dong, Z.-G., Ni, P., Zhu, J., Yin, X. \& Zhang, X. Toroidal dipole response in a multifold double-ring metamaterial. Opt. Express 20, 13065-13070 (2012).

57. Ye, Q. W. et al. The magnetic toroidal dipole in steric metamaterial for permittivity sensor application. Phys. Scr. 88, (2013).

58. Fan, Y., Wei, Z., Li, H., Chen, H. \& Soukoulis, C. M. Low-loss and high-Q planar metamaterial with toroidal moment. Phys. Rev. B 87, 115417 (2013).

59. Savinov, V., Delfanazari, K., Fedotov, V. A. \& Zheludev, N. I. Planar Superconducting Toroidal Metamaterial: A Source for Oscillating Vector-Potential? in 2014 Conference on Lasers and Electro-Optics (CLEO) (2014).

60. Ding, C. et al. Stable terahertz toroidal dipolar resonance in a planar metamaterial. Phys. Status Solidi 252, 13881393 (2015).

61. Huang, Y. W. et al. Design of plasmonic toroidal metamaterials at optical frequencies. Opt. Express 20, 1760-1768 (2012).

62. Wu, P. C. et al. Three-dimensional metamaterials: from split ring resonator to toroidal metamolecule. in Proc. SPIE 9163, Plasmonics: Metallic Nanostructures and Their Optical Properties 91630M (2014). 
63. Dong, Z.-G. et al. Optical toroidal dipolar response by an asymmetric double-bar metamaterial. Appl. Phys. Lett. 101, 144105 (2012).

64. Dong, Z.-G. et al. All-optical Hall effect by the dynamic toroidal moment in a cavity-based metamaterial. Phys. Rev. B 87, 245429 (2013).

65. Zhang, Q., Xiao, J. J. \& Wang, S. L. Optical characteristics associated with magnetic resonance in toroidal metamaterials of vertically coupled plasmonic nanodisks. J. Opt. Soc. Am. B 31, 1103-1108 (2014).

66. Liu, W., Zhang, J. \& Miroshnichenko, A. E. Toroidal dipole induced transparency in core-shell nanoparticles. Laser Photon. Rev. 9, 564-570 (2015).

67. Kim, S.-H. et al. Subwavelength localization and toroidal dipole moment of spoof surface plasmon polaritons. Phys. Rev. B 91, 035116 (2015).

68. Li, J. et al. Excitation of plasmon toroidal mode at optical frequencies by angle-resolved reflection. Opt. Lett. 39, 6683-6686 (2014).

69. Ögüt, B., Talebi, N., Vogelgesang, R., Sigle, W. \& van Aken, P. A. Toroidal Plasmonic Eigenmodes in Oligomer Nanocavities for the Visible. Nano Lett. 12, 5239-5244 (2012).

70. Huang, Y. W. et al. Toroidal lasing spaser. Sci. Rep. 3, 1237 (2013).

71. Basharin, A. A. et al. Dielectric Metamaterials with Toroidal Dipolar Response. Phys. Rev. X 5, 011036 (2015).

72. Vinogradov, A. P. \& Aivazyan, A. V. Scaling theory for homogenization of the Maxwell equations. Phys. Rev. E 60, 987-993 (1999).

73. Fedotov, V. A., Rogacheva, A. V., Savinov, V., Tsai, D. P. \& Zheludev, N. I. Resonant Transparency and NonTrivial Non-Radiating Excitations in Toroidal Metamaterials. Sci. Rep. 3, 2967 (2013).

74. Liu, W., Zhang, J., Lei, B., Hu, H. \& Miroshnichenko, A. E. Invisible nanowires with interfering electric and toroidal dipoles. Opt. Lett. 40, 2293-2296 (2015).

75. Boardman, A. D. \& Marinov, K. Nonradiating and radiating configurations driven by left-handed metamaterials. $J$. Opt. Soc. Am. B 23, 543-552 (2006).

76. Marengo, E. A. \& Ziolkowski, R. W. Nonradiating sources, the Aharonov-Bohm effect, and the question of measurability of electromagnetic potentials. Radio Sci. 37, 10-19 (2002).

77. Zagoskin, A. M., Chipouline, A., Il'ichev, E., Johansson, J. R. \& Nori, F. Toroidal qubits: naturally-decoupled quiet artificial atoms. arXiv:1406.7678 [cond-mat, physics:quant-ph] (2014).

78. Raybould, T. A. et al. Toroidal optical activity. arXiv:1508.06192 [physics.optics] (2015).

79. Hellwarth, R. W. \& Nouchi, P. Focused one-cycle electromagnetic pulses. Phys. Rev. E 54, 8

80. N. I . Zheludev et al. Propagating and localized toroidal excitations in free space and metamaterials. in SPIE Optics \& Photonics (2015).

81. Raybould, T. A., Fedotov, V. A., Papasimakis, N., Youngs, I. J. \& Zheludev, N. I. Focused electromagnetic doughnut pulses and their interaction with interfaces and nanostructures. 11 (2015). at $<$ http://arxiv.org/abs/1512.04285>

82. Ziolkowski, R. W. Localized transmission of electromagnetic energy. Phys. Rev. A 39, 2005-2033 (1989).

83. Ziolkowski, R. W. Properties of electromagnetic beams generated by ultra-wide bandwidth pulse-driven arrays. IEEE Trans. Antennas Propag. 40, 888-905 (1992).

84. Lemak, S. et al. Toroidal Structure and DNA Cleavage by the CRISPR-Associated [4Fe-4S] Cluster Containing Cas4 Nuclease SSO0001 from Sulfolobus solfataricus. J. Am. Chem. Soc. 135, 17476-17487 (2013).

85. Watson, D. W., Jenkins, S. D., Ruostekoski, J., Fedotov, V. A. \& Zheludev, N. I. Toroidal dipole excitations in metamolecules formed by interacting plasmonic nanorods. arXiv:1510.05609 [physics.optics] (2015).

86. Bao, Y., Zhu, X. \& Fang, Z. Plasmonic Toroidal Dipolar Response under Radially Polarized Excitation. Sci. Rep. 5, 11793 (2015).

87. No Title. at <http://www.interactive-biology.com/3945/production-and-regulation-of-rbcs/>

88. Thorner, G., Kiat, J.-M., Bogicevic, C. \& Kornev, I. Axial hypertoroidal moment in a ferroelectric nanotorus: A way to switch local polarization. Phys. Rev. B 89, 220103 (2014).

89. Leroy, B. How to convert the equations of electromagnetism from Gaussian to SI units in less than no time. Am. $J$. Phys. 53, 589-590 (1985). 


\section{Figure Legends}

FIG. 1. Toroidal structures at different length scales. Toroidal topology, encountered very often in both artificial and naturally occurring objects, provides an indication for the presence of spontaneous or induced toroidal moments. Top row, from left to right: solenoidal currents lead to a toroidal moment in the atomic nucleus ${ }^{17}$; quaternary structure of archaeon (S. solfataricus Cas4) protein ${ }^{84}$; red blood cells take a biconcave, torus-like shape ${ }^{87}$. Bottom row from left to right: benzene (left), hexaphenylbenzene (centre) and toroidal carbon cage consisting of 120 carbon atoms $^{8}$ (right) are organic molecules with elements of toroidal symmetry; perovskite $\left(\mathrm{BaTiO}_{3}\right)$ nanotori ${ }^{88}$ are examples of artificial toroidal structures.

FIG. 2. The "Multipole Zoo" (adapted from ${ }^{51}$ ). Electric multipoles represent charge configurations (far left column), whereas magnetic multipoles correspond to current sources (second column from left). The (magnetic) toroidal multipole family (second column from the right) corresponds to current distributions that cannot be represented by electric and magnetic multipoles. Same order members of each multipole family have identical power radiation patterns of corresponding oscillating multipoles (far right column). Electric and toroidal dipoles have also identical radiated field patterns as indicated by the same colour (red) arrows.

FIG. 3. Toroidal metamaterials. (a) Artistic drawing of the metamaterial unit cell used for the first demonstration of a dynamic toroidal dipole absorption resonance ${ }^{55}$. (b) Planar low-loss split-ring metamaterial on a dielectric substrate supports toroidal modes of excitation $^{58}$. (c) A scaled-down version of the metamaterial presented in (a) shows plasmonic toroidal response at optical wavelengths ${ }^{62}$. (d) Optical toroidal metamaterial exploiting resonant plasmonic response $^{63}$. (e) Spoof plasmon structure supports a toroidal dipole excitation at oblique angles of incidence $^{68}$. (f) Plasmonic oligomers consisting of voids in metallic films exhibit toroidal response at visible wavelengths and can be excited by a free-electron beam ${ }^{69}$. (g) Low-loss toroidal metamaterial consisting of dielectric cylinders ${ }^{71}$. (h) Interference of induced electric and toroidal dipoles in a resonantly transparent metamaterial consisting of dumbbell-shaped apertures, leads to a non-radiating configuration ${ }^{73}$. (i) Near-field signature of toroidal dipole excitation in a dielectric nanoparticle ${ }^{49}$.

FIG. 4. Non-radiating configurations consist of a toroidal dipole, represented by a solenoid with oscillating poloidal currents, and an electric dipole, represented by a pair of opposite charges, oscillating on the same frequency as the currents. With an appropriate phase difference and amplitudes of their oscillations destructive interference takes place: the combined source does not radiate electromagnetic fields. However, the scalar $(\phi)$ and vector (A) potentials associated with radiation of these dipoles do not cancel, but instead propagate to the farfield. Hence, a non-radiating configuration acts as a source of electromagnetic potentials (but not electromagnetic fields). The physical significance and detectability of these potentials are not established and are being actively discussed in the literature.

FIG. 5. "Focused Doughnut" (FD) pulses. Artistic representation of a transverse magnetic (TM) focused doughnut pulse propagating from right to left. Here, the magnetic field is azimuthally polarized and confined in a torus-shaped region, and the electric field is winding along the meridians of the torus resulting in a longitudinal component at the centre of the pulse. Focused doughnut pulses have broad spectrum and are characterized by two parameters, $\mathrm{q}_{1}$, which represents an effective wavelength, and $\mathrm{q}_{2}$, which quantifies the focal depth and is analogous to the Rayleigh range of conventional beam optics. The projected cross-section demonstrates the confinement of the pulse energy in two adjacent toroidal regions, while the white arrow indicates the propagation direction. 


\section{Tables}

\begin{tabular}{|c|c|c|c|}
\hline $\begin{array}{l}\text { Multipole } \\
\text { family }\end{array}$ & Dipole Moment & $\begin{array}{c}\text { Interaction energy } \\
W=\int d^{3} r\left(\rho \varphi-\frac{1}{c} \boldsymbol{J} \cdot \boldsymbol{A}\right)\end{array}$ & $\begin{array}{c}\text { Far-field radiation } \\
\text { patterns } \\
(\hat{\boldsymbol{r}} \cdot \boldsymbol{S}) \\
\end{array}$ \\
\hline Electric & $\boldsymbol{p}=\frac{1}{i \omega} \int d^{3} r \boldsymbol{J}$ & $-\boldsymbol{p} \cdot \boldsymbol{E}-\frac{1}{c} \frac{\partial}{\partial t}(\boldsymbol{p} \cdot \boldsymbol{A})$ & $\frac{\omega^{4}}{4 \pi c^{3}}|\boldsymbol{p}|^{2}\left(1-(\hat{\boldsymbol{r}} \cdot \widehat{\boldsymbol{p}})^{2}\right)$ \\
\hline Magnetic & $\boldsymbol{m}=\frac{1}{2 c} \int d^{3} r(\boldsymbol{r} \times \boldsymbol{J})$ & $-m \cdot B$ & $\frac{\omega^{4}}{4 \pi c^{3}}|\boldsymbol{m}|^{2}\left(1-(\hat{\boldsymbol{r}} \cdot \widehat{\boldsymbol{m}})^{2}\right)$ \\
\hline Toroidal & $\boldsymbol{T}=\frac{1}{10 c} \int d^{3} r\left[\boldsymbol{r}(\boldsymbol{r} \cdot \boldsymbol{J})-2 r^{2} \boldsymbol{J}\right]$ & $-\boldsymbol{T} \cdot(\dot{\boldsymbol{D}}+4 \pi \boldsymbol{J}) / c$ & $\frac{\omega^{6}}{4 \pi c^{5}}|\boldsymbol{T}|^{2}\left(1-(\hat{\boldsymbol{r}} \cdot \widehat{\boldsymbol{T}})^{2}\right)$ \\
\hline
\end{tabular}

Table 1. Dipole moments $(\boldsymbol{p}, \boldsymbol{m}, \boldsymbol{T})$, interaction energy $(W)$ with an electromagnetic field, and radiated power (expressed as the radial component of the Poynting vector $\boldsymbol{S}$ ) for the three multipole families (electric, magnetic, toroidal). 
Nuclear Toroidal

Moment

Nuclear

Proteins

Microscopic Life

Current
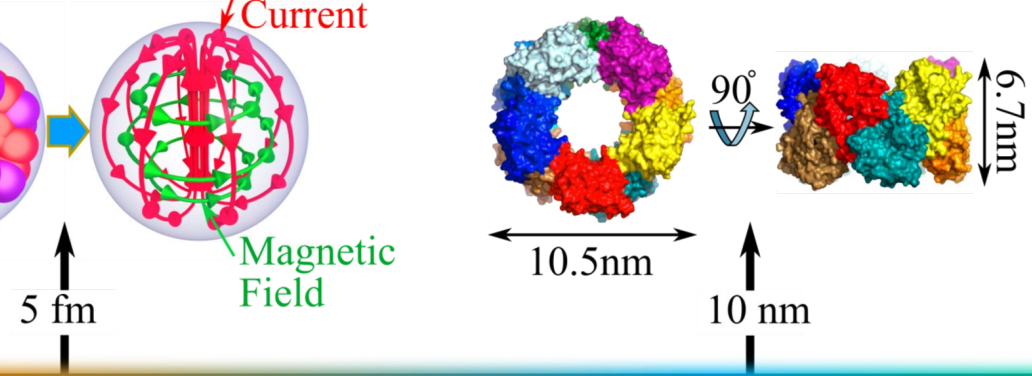

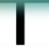

$1 \mathrm{~nm}$

$\downarrow$

Organic Molecules
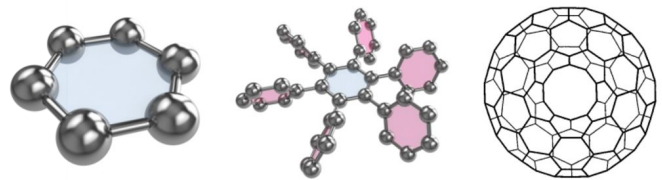

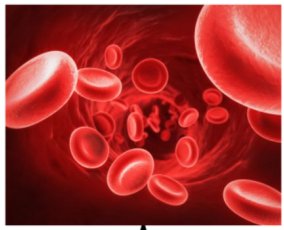

$1 \mu \mathrm{m}^{+}$

I

Size

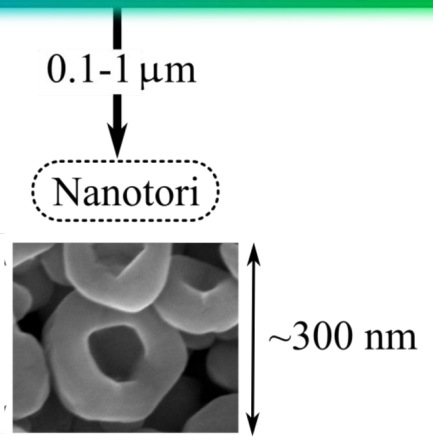


Electric Multipoles

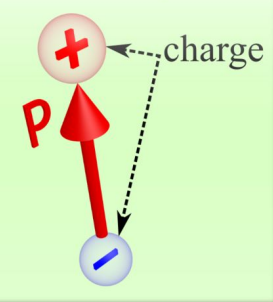

$Q^{(e)}$
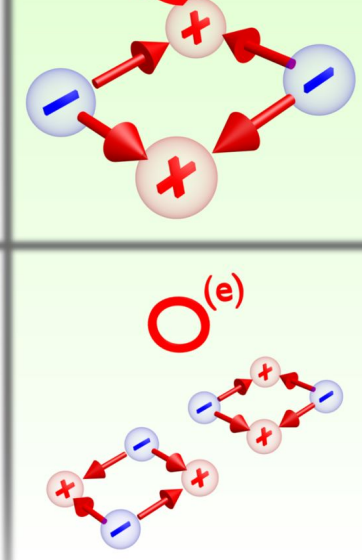

Magnetic Multipoles

current

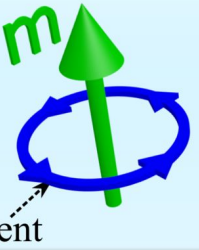

$\mathrm{O}^{(\mathrm{m})}$
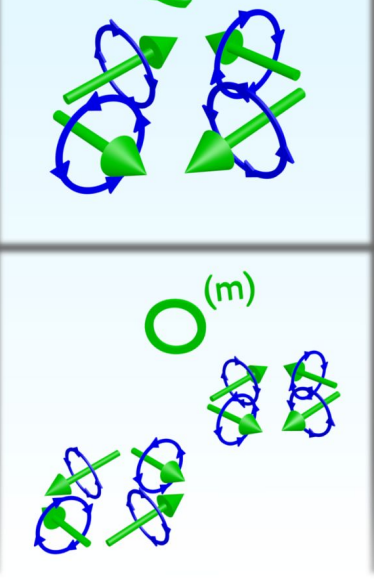
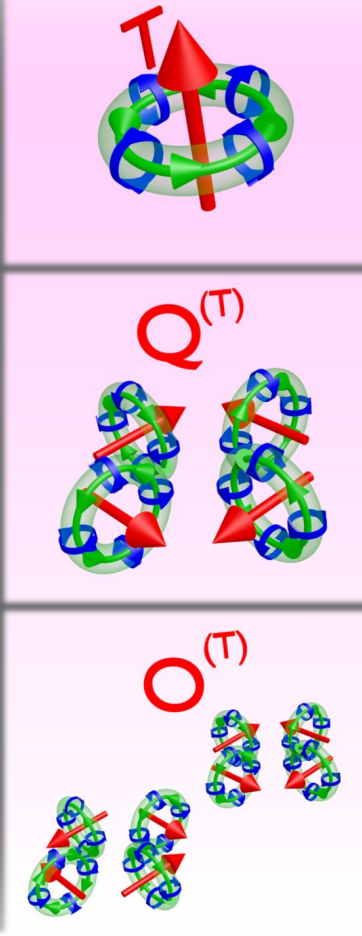
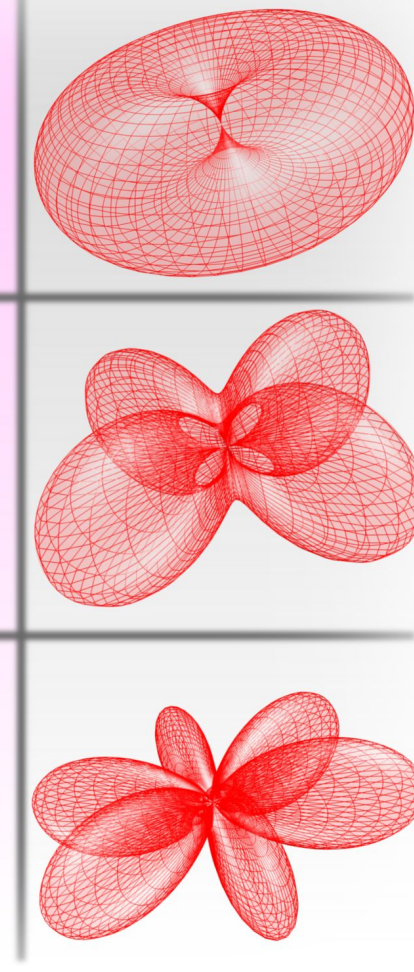


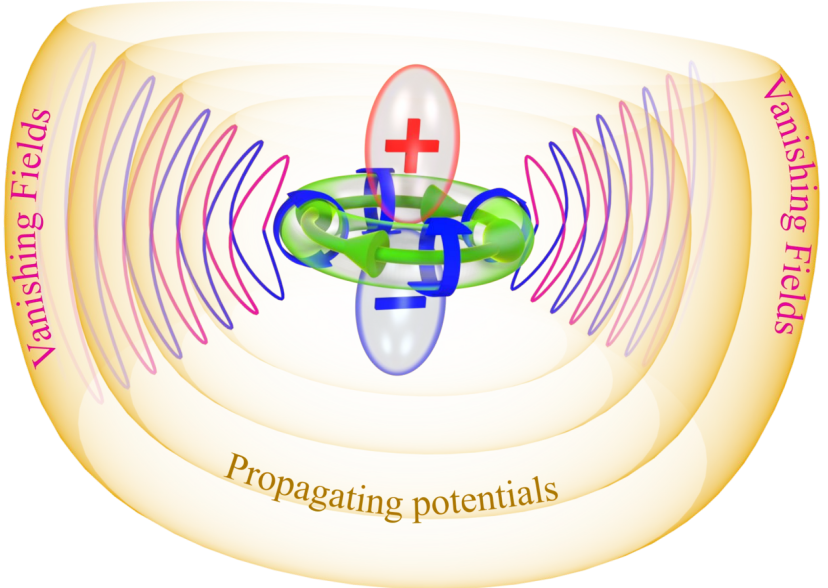




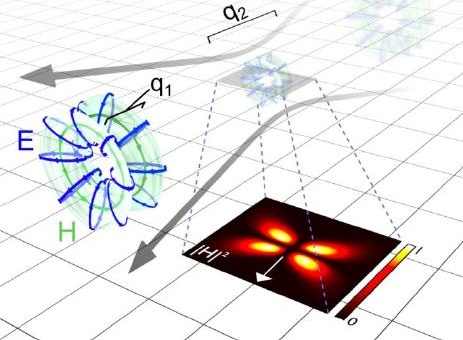

